\title{
Aprendizaje de Redes de Computadores mediante el uso de Proyectos en una Titulación de Videojuegos Learning Computer Networks through the use of Projects in a Video Game degree
}

\author{
Mercedes Fernández Redondo, Carlos Hernández Espinosa, Jorge Sales Gil \\ redondo@uji.es, espinosa@uji.es, salesj@uji.es \\ Departamento de Ingeniería y Ciencia de los Computadores \\ Universitat Jaume I \\ Castellón, España
}

\begin{abstract}
Resumen- En este trabajo se presenta una experiencia docente que utiliza metodología de aprendizaje basada en proyectos desarrollada en la asignatura "Redes y Sistemas Multijugador" de tercer curso del Grado de Diseño y Desarrollo de Videojuegos. El objetivo fundamental es hacer ver a los alumnos la importancia de las redes al programar videojuegos, así como potenciar el aprendizaje y la adquisición de competencias básicas de la asignatura. El segundo año en que se impartió docencia de la asignatura se introdujo la realización voluntaria de un proyecto en el que programaban en java un juego básico en red usando directamente las primitivas de comunicación de sockets estudiadas en redes. La experiencia fue positiva. Por ello, en este artículo se describen la forma de introducir dicho proyecto en el aprendizaje y evaluación de la asignatura, establecer mecanismos de autocorrección de los proyectos (rúbricas) y realizar periódicamente encuestas a los alumnos para medir la satisfacción de su aprendizaje en la asignatura.
\end{abstract}

Palabras clave: proyectos, sistemas multijugador, sockets, juegos en red.

\begin{abstract}
This work focuses on a project-based learning teaching methodology experience that has been recently developed in the context of a Videogame Design and Development degree. The main objective is that students become aware of the importance of networks in the context of videogame programming, and also to promote the learning and acquisition of the basic competences related to the computer networks subject. During the second year of teaching this subject, we promoted the development of a project in which students had to develop a simple java game with networking capabilities. The experience resulted in positive outcomes. As a result, the article describes the way of introducing the aforementioned project in the learning process and evaluation of the course, how to establish auto correction mechanisms for the projects (rubrics) and how to perform periodical surveys to the students in order to assess their learning satisfaction degree during the course.
\end{abstract}

Keywords: projects, multiplayers systems, sockets, networks games.

\section{INTRODUCCIÓN}

En cursos anteriores los autores de este artículo desarrollamos diversos proyectos de mejora docente con aplicación en la docencia de asignaturas de las áreas de Redes de Computadoras y Sistemas Operativos. Así, publicamos varios artículos con nuestros resultados [4-7]. En los dos últimos años las mejoras se han basado principalmente en aplicar aprendizaje basado en problemas y proyectos.

En publicaciones de otros autores $[9,11]$ se indica que el profesor debe servir solo como apoyo a la labor realizada por el alumno en el proyecto, pero debe ser este, el propio alumno, el que resuelva las situaciones de conflicto. Se debe evitar el exceso de guía que lleve a que los alumnos no realicen el aprendizaje deseado.

Además, el trabajo basado en problemas y proyectos requiere trabajo en grupo. Se pretende que el aprendizaje no sea individual sino en grupo. Así, en [2] se describen algunas estrategias basadas en la combinación de evaluación individual y en grupo, utilizadas para mejorar la implicación de los miembros de un equipo en el trabajo que realicen conjuntamente. También es importante saber el tipo de conflictos que pueden surgir dentro del equipo cuando se realiza un trabajo en grupo, $[1,3,10]$.

Nosotros hemos observado que el aprendizaje basado en problemas y proyectos en el cual se trabaja casi diariamente en la asignatura, lleva a mayor motivación del alumno que a su vez concluye en un mejor rendimiento académico.

Por ello, pensamos que para el aprendizaje de una parte de nuestra asignatura se podría introducir el desarrollo de proyectos. No se trata totalmente de una experiencia ABP ya que, aunque sí que se eliminan las clases teóricas, solo se hace para un único tema de la asignatura, que por otra parte es el más práctico y el más relacionado con los temas de la titulación (desarrollo de videojuegos en red). Además, el desarrollo del proyecto sirve como elemento integrador de todos los conceptos aprendidos sobre redes a lo largo del curso aplicándolo así a un desarrollo práctico supervisado por el profesor.

\section{CONTEXTO}

En el curso 2014-2015 se inicia la docencia de la asignatura Redes y Sistemas Multijugador de tercer curso del Grado de Diseño y Desarrollo de Videojuegos. 
Se trata de una asignatura en la que se estudian aspectos básicos de redes para posteriormente ver su utilización en el diseño de juegos en red.

Sin embargo, debido principalmente al nombre de la asignatura Redes y Sistemas Multijugador, las expectativas de los alumnos eran otras, y las preguntas que hacían a final de curso eran ¿Dónde hemos dado sistemas multijugador? ¿Para qué quiere un diseñador de juegos saber redes?

Realmente los alumnos sí habían trabajado estos temas, pero no eran conscientes de ello, no los asociaban con el diseño y desarrollo de juegos que habían dado en otras asignaturas. Ahora tenían que incluir en la programación de un juego, la conexión en red entre las máquinas que juegan.

En clase de laboratorio habían programado en java un cliente y un servidor concurrente que admitía conexiones desde un navegador de internet. Pero los alumnos no vieron que se trataba de un programa cliente que podría ser alguien que se conecta a un servidor, manda jugadas y recibe estados de juego y un programa servidor que mantiene el juego y atiende a diferentes clientes (sistema multijugador).

Creímos que era necesario que trabajaran precisamente este aspecto y pensamos que era interesante plantearles para ello un proyecto. Así les mandamos diseñar un juego, pero sin la ayuda de los módulos y herramientas a las que estaban acostumbrados. En nuestra asignatura lo importante es que es un juego en red y se trata pues de desarrollar precisamente eso, la comunicación en red entre entidades, en este caso entre jugadores o entre jugadores y servidores de juegos en red.

En el pasado curso 2015-2016 como prueba de esta estrategia se les planteó a los alumnos de forma voluntaria la realización de un proyecto en grupo de dos en el cual programaban en java un juego básico en red usando directamente las primitivas de comunicación de sockets estudiadas en redes. En las clases teóricas que correspondían a los contenidos relacionados con la programación de sockets (tema3 y tema4) se explicaron brevemente los métodos de comunicación en red entre dos entidades y se indicó de que forma obtener información para realizar su proyecto. Se impartieron solamente 3 horas de clases teóricas y los contenidos no vistos debían trabajarlos los alumnos al desarrollar su proyecto.

El resultado fue bastante satisfactorio, los alumnos entendieron como aplicar redes al diseño de videojuegos y por ello, en el presente curso se han realizado modificaciones en la metodología y evaluación para incluir la realización de este proyecto de forma obligatoria.

En adelante, describiremos la problemática inicial, que objetivos nos planteamos, de qué manera afecta a la metodología y evaluación de la asignatura y algunos resultados y trabajos futuros.

El objetivo principal es mejorar la asignatura de "Redes y Sistemas Multijugador" del segundo semestre de tercer curso del Grado de Diseño y Desarrollo de Videojuegos.

Se trata de la única asignatura de la titulación en la que estudian redes de computadores. Teniendo en cuenta las competencias y resultados de aprendizaje había que incluir en el temario aspectos sobre la utilización de protocolos de comunicación básicos para el desarrollo de aplicaciones en red, así como la problemática de la programación paralela, concurrente, distribuida y en tiempo real para el diseño de juegos en red multijugador.

Esto último, se imparte en el primer semestre en la asignatura "Sistemas Operativos" de tercero de la titulación. Nosotros lo que hacemos es tener en cuenta los conceptos estudiados en ella a la hora de programar la comunicación en los juegos.

\section{DESCRIPCIÓN}

Teniendo en cuenta los contenidos de la asignatura vimos que por un lado había que impartir conceptos básicos de redes y por otro tenían que estudiar de que manera "aplicar redes en la programación de juegos”. Así decimos que esta segunda parte convenía trabajarla como proyectos desarrollados en grupo.

Así, el objetivo principal requería: un cambio en la organización docente (orden de impartición de temas), cambios en la realización de actividades del alumno (realización de test, problemas y proyectos) y cambios en el sistema final de evaluación.

En adelante vamos a describir las actividades y recursos utilizados para la consecución de estos objetivos.

\section{A. Unidades temáticas iniciales}

Durante el pasado curso, el temario de la asignatura "Redes y Sistemas Multijugador” se organizó en dos bloques:

- $\quad$ Bloque 1: Conceptos de Redes.

- Bloque 2: Sistemas Multijugador.

En el primer bloque, se trataba el diseño de una red por capas y se explicaban los conceptos básicos de redes que considerábamos debía conocer un diseñador de juegos. En el laboratorio hacían prácticas de configuración de redes con un simulador.

En el segundo bloque se introducía la programación en java de aplicaciones en red con socket. En el laboratorio se dedicaban 4 sesiones para la programación en java de un cliente y un servidor en red y la comunicación entre ellos.

A pesar de haber introducido la programación con socket para comunicación de procesos en redes, los alumnos no tenían claro donde habíamos dado el tema de "Sistemas Multijugador” ya que no habían utilizado estos conceptos en la programación de un juego.

\section{B. Cambios en la metodología docente y evaluación}

Por ello, como hemos dicho antes, propusimos a los alumnos que voluntariamente trabajaran en grupos de dos, en el desarrollo de un juego en red básico. Esto requiere la programación de los procesos cliente y servidor (que ya hacían en el laboratorio), la programación de la conexión entre ellos (entre máquinas remotas) y el propio juego (tres en raya, ahorcado, barcos).

Entre los esquemas de conexión (que explicábamos en teoría) dejamos que escogieran entre, el modelo de servidor (clientes se conectan a un servidor que es el que tiene el juego) o el modelo cliente P2P (dos clientes se conectan entre sí para jugar por lo que ambos deben tener el juego). 
La experiencia fue muy buena, de 24 grupos totales que había en clase, 15 acabaron el juego y 5 de ellos obtuvieron la puntuación máxima. Casi todos escogieron el modelo de servidor ya que es más sencillo.

En el presente curso 2016-2017 hemos introducido un cambio en la metodología y evaluación de la asignatura. Se ha modificado el temario realizando un cambio en el orden de los temas. El temario final ha sido:

- Tema 1. Introducción a las redes informáticas.

- Tema 2. La capa de red

- Tema 3. La capa de transporte. Programación de sockets.

- Tema 4. La capa de aplicación.

- Tema 5. La capa de enlace de datos.

- Tema 6. Aspectos de seguridad.

Los aspectos relativos a sistemas distribuidos, concurrencia, etc., estaría incluidos en la programación de sockets, tema 3.

En la evaluación continua mantuvimos las actividades existentes en cursos anteriores: test de evaluación parcial, realización de problemas en casa, realización de prácticas en el laboratorio y añadimos la realización obligatoria en grupos de 2 alumnos (los mismos del laboratorio) de un proyecto para realizar de forma "no presencial”. Se sustituye con este último, gran parte de la docencia presencial de los temas 3 y 4 de los que solo tendrán 3 horas presenciales.

Con el cambio de temario, pudimos proponer el proyecto una vez impartido los temas 3 y 4 (3 horas presenciales) por lo que los alumnos dispusieron de casi 3 meses para su realización y entrega. Además, ya habían realizado en el laboratorio la programación de un cliente y un servidor que les fue de bastante ayuda.

Así, el peso de las actividades en la evaluación sería:

- $\quad$ Test y problemas $15 \%$

- Actividades de laboratorio presenciales $25 \%$

- Realización Proyecto no presencial $10 \%$

- Examen final 50\%

Al ser una asignatura del segundo semestre hemos podido ya realizar todos los cambios.

\section{Aprendizaje mediante proyectos}

La introducción del proyecto de forma obligatoria se debe a que pensamos que con las modificaciones temporales del temario hay mucho tiempo para realizarlo. Por eso, se exige la programación de los dos modelos de comunicación, clienteservidor y $\mathrm{P} 2 \mathrm{P}$.

El curso pasado el proyecto se propuso muy tarde, un mes antes de acabar las clases, por lo que los alumnos se quejaron de que estaba muy ocupados con otras asignaturas por lo que no tenían tiempo de acabarlo satisfactoriamente. Solo 15 de 24 grupos lo acabaron, pero solo a 5 grupos les funcionó completamente.

Aunque el peso del proyecto es pequeño no implica una gran dificultad y pensamos que es de gran ayuda para la comprensión final de la asignatura ya que aprenden cuales son los aspectos a tener en cuenta al usar una red: como el direccionamiento (tema2), como establecer la comunicación entre dos entidades (tema 3,5,6), y como desarrollar un juego en red sencillo (tema4). Es decir, cubren de forma práctica todo el temario del curso.

\section{Otras herramientas}

Puesto que el proyecto tiene un plazo de entrega grande para impedir el abandono hasta final de curso, es decir, intentar que los alumnos trabajen desde el día que se les propone, se quería poner a disposición de los alumnos alguna herramienta de autoevaluación y establecer plazos de consecución de objetivos.

Se pensó que una herramienta adecuada podía ser la rúbrica, en la que se establecieran diversos objetivos que habría que ir alcanzando en el proyecto en una fecha determinada. Estas rúbricas servirían a los alumnos de guía para ir desarrollando el proyecto, aunque no se usarían realmente para la calificación final que sería al criterio del profesor.

Finalmente se establecieron dos fechas de entrega, una para el algoritmo cliente-servidor (última semana de clase) y otra para el algoritmo P2P (15 días después). En el documento de presentación del proyecto se fijaron como objetivos los siguientes puntos:

- Desarrollo del algoritmo cliente-servidor.

- $\quad$ Programación de este algoritmo.

- Pruebas de conexión hasta que funcione.

- Desarrollo del juego en el cliente y en el servidor.

- $\quad$ Pruebas finales de funcionamiento.

- Redacción de un documento explicativo del trabajo realizado y que explique el funcionamiento del juego.

- Desarrollo del algoritmo P2P.

- $\quad$ Programación de este algoritmo.

- Pruebas de conexión hasta que funcione.

- Desarrollo del juego en un cliente.

- $\quad$ Pruebas finales de funcionamiento.

- Redacción de un documento explicativo del trabajo realizado y que explique el funcionamiento del juego.

Estos puntos son los que pretendíamos utilizar para elaborar la rúbrica, aunque al final no se utilizó por falta de tiempo.

Aunque en este curso no se han realizado, consideramos de gran importancia consultar a los alumnos de forma anónima a lo largo del curso para ver el éxito de los cambios que se van introduciendo en la asignatura.

Para el próximo curso se confeccionarán test_online que podrán realizar los alumnos de forma voluntaria y confidencial. Las preguntas deben reflejar cosas como el grado de satisfacción con las actividades realizadas, si la duración es adecuada y si consideran que la actividad mejora su rendimiento y comprensión. Estos test se realizarán:

- $\quad \mathrm{Al}$ acabar ciertas sesiones de laboratorio, al finalizar las actividades correspondientes a cada tema (incluirá unas tres o cuatro sesiones de laboratorio).

- Después de realizar las actividades de test y problemas de los bloques de temas 2, 3-4 y 5-6.

- $\quad \mathrm{Al}$ entregar el proyecto.

Algunas preguntas podrían ser: ¿Qué tiempo has dedicado a la actividad? ¿has encontrado suficiente información en el 
material de clase? ¿habrías añadido o quitado algo de la actividad? ¿el tiempo ha sido suficiente? ...etc.

Durante este curso hemos recibido algunos comentarios por parte de los alumnos y llegamos a la conclusión de que la realización de proyecto es positiva y que les ha motivado en el aprendizaje de redes ya que para desarrollar su juego tenían la necesidad de conocer ciertos conceptos básicos de comunicación en red.

\section{RESUltados}

El grado de Diseño y Desarrollo de Videojuegos se implantó en el curso académico 2012-2013. La asignatura Redes y Sistemas Multijugador, cuya docencia queremos mejorar en este trabajo, es del segundo semestre del tercer curso y se comenzó a impartir en febrero de 2015, en el curso 2014-2015. Es pues, una asignatura con tan solo 3 años de docencia.

Para evaluar los resultados de los cambios docentes propuestos en ella se deben analizar diversos aspectos a lo largo y al final de curso. Entre ellos, los que consideramos más importantes son:

- Los índices de participación activa en la asignatura (asistencia a clase).

- Implicación de los alumnos a la hora de realizar los trabajos propuestos (motivación).

- Índices de participación en las evaluaciones de teoría y problemas.

- Evolución de los alumnos a lo largo del curso.

- Por último, consecución de los objetivos planteados (superación de la asignatura).

Consideramos que lo más importante son los datos de la evaluación continua ya que si durante ella los alumnos han conseguido adquirir las competencias de la asignatura, también conseguirán realizar el examen final con éxito. Esta opinión es compartida por otros docentes como podemos ver en [8].

Pretendemos que los alumnos trabajen semanalmente en la asignatura y para ello les proponemos la realización individual de test y problemas de cada unidad que entregan al profesor al pasar un cierto tiempo (antes de comenzar el siguiente tema). Los llamamos test y problemas de casa. También en grupos de 2 alumnos, realizan cada semana en el laboratorio actividades relacionadas con la teoría impartida.

Todo esto motivaba a los alumnos a asistir a clase para poder seguir “día a día” la asignatura y tener una evolución progresiva en el aprendizaje. Si no se asiste a clase el esfuerzo será mayor ya que el alumno debía de realizarlo por sí mismo, sin ayuda de nadie.

Los profesores realizan retroalimentación de las actividades realizadas por los alumnos mediante la corrección de ellas con el objetivo de que los alumnos puedan completar su aprendizaje corrigiendo sus errores o resolviendo sus dudas.

La asignatura desde siempre ha tenido un alto grado de asistencia a clase. Los profesores han pasado en todas sus clases una hoja de firmas para controlar la asistencia, aunque solo a nivel informativo, ya que la asistencia "no es obligatoria”. A clase de teoría asisten alrededor de un 95\% de los alumnos y al laboratorio todos.

Además, el índice de aprobados en los tres años de docencia que lleva la asignatura es altísimo.

- En el curso 2014-2015 eran 38 alumnos matriculados. Todos realizaron las pruebas de evaluación continua. Todos se presentaron al examen final y suspendieron 3 alumnos. Estos se presentaron al examen en segunda convocatoria y aprobaron. Este curso hubo un 100\% de aprobados. De 38 alumnos, 10 tuvieron más nota en el examen que en la evaluación continua y 28 nota inferior.

- En el curso 2015-2016 eran 46 alumnos matriculados. Realizaron la evaluación continua satisfactoriamente 44 . Un alumno no la completó y otro no la hizo. Se presentaron al examen final 45 alumnos y aprobaron 44 (los que habían realizado la evaluación continua). En la segunda convocaría había 2 alumnos que no se presentaron al examen final. Aprobaron el 95.7\%. De los 44 alumnos, 32 tuvieron más nota en el examen que en la evaluación continua y 12 nota inferior.

- En el curso 2016-2017 había 50 alumnos matriculados. Se presentaron al examen final 47 alumnos y aprobaron 45. En la segunda convocaría aprobaron los otros 2 alumnos. Aprobaron el 94\%. De los 47 alumnos, 27 tuvieron más nota en el examen que en la evaluación continua y 20 nota inferior.

En la figura 1 puede verse el porcentaje de aprobados de los tres cursos de docencia de la asignatura. Vemos que es muy similar y que no hay suspensos, los alumnos que realizan la evaluación continua y van al examen aprueban.

El índice de no presentados son alumnos que no han realizado la mayoría de las actividades de evaluación continua y que al final no se presentaron al examen final.

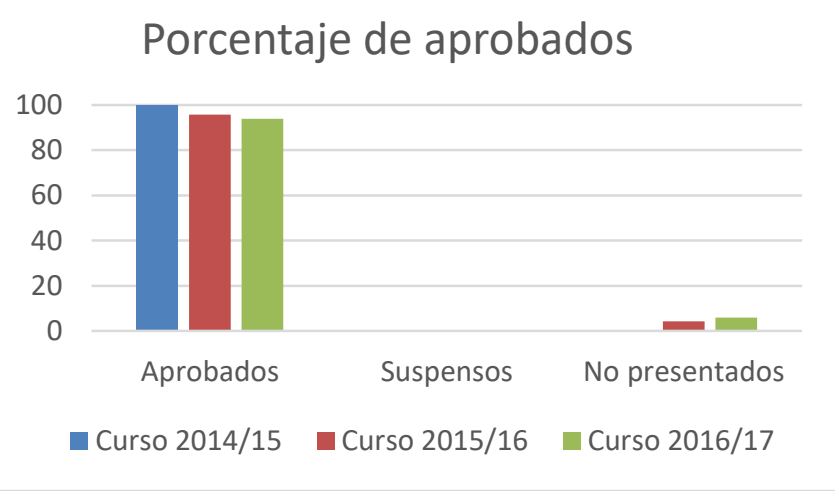

Figura 1: Porcentaje de aprobados por curso.

Como dijimos antes, como parte de la evaluación continua se ha propuesto en los dos últimos años la realización de un proyecto (programación de un juego simple en red) que englobaba el uso de gran parte de los conocimientos que se van adquiriendo durante el curso. 
En el curso 2015-2016 la realización fue voluntaria y se dejó que los alumnos escogieran uno de los métodos de comunicación cliente-servidor o P2P.

En el presente curso 2016-2017 el proyecto era obligatorio y tenían que programar los dos métodos. La razón es que veíamos interesante que conocieran las dos metodologías y la mejor forma era intentar programarlas.

Durante el presente curso todos los alumnos debían realizar obligatoriamente el proyecto. Se utilizaron grupos de dos alumnos, los mismos del laboratorio. De los 25 grupos de clase, 2 no realizaron los proyectos. Del resto, 14 grupos obtuvieron una nota superior a 7 y 9 grupos obtuvieron menos de 5. Sin embargo, de estos 9 últimos, 4 no realizaron uno de los dos modelos y tenían algún error en el otro y 5 tenían casi todo mal.

Como dijimos el $50 \%$ de la nota de la asignatura se debe a la evaluación continua y el otro $50 \%$ al examen final. Si comparamos las notas de la evaluación continua con las obtenidas en el examen final son muy similares.

Sin embargo, el primer año en el que no se propuso el proyecto solo 10 alumnos de 38 obtuvieron más nota en el examen que en la evaluación continua. En el segundo año que se propuso el proyecto (aunque voluntario) fueron 32 alumnos de 44 los que obtuvieron más nota en el examen final.

En la figura 2 se puede observar la comparación del número de alumnos que obtuvieron más nota en el examen final que en la evaluación continua en cada uno de los cursos. Durante el primer curso que no se ofreció la realización del proyecto un $26,32 \%$ del total de alumnos obtuvo más nota en el examen final.

Sin embargo, en los dos siguientes cursos el porcentaje crece, en el curso 2015-16 es un 69\% y en el presente curso 2016-17 es de un 54\%.

Esto nos lleva a pensar que efectivamente si durante la evaluación continua se han adquirido las competencias de la asignatura en el examen final se obtiene el mismo resultado o mejor y que la realización del proyecto ayuda a la comprensión de la asignatura, más alumnos obtienen mejor nota en el examen final.

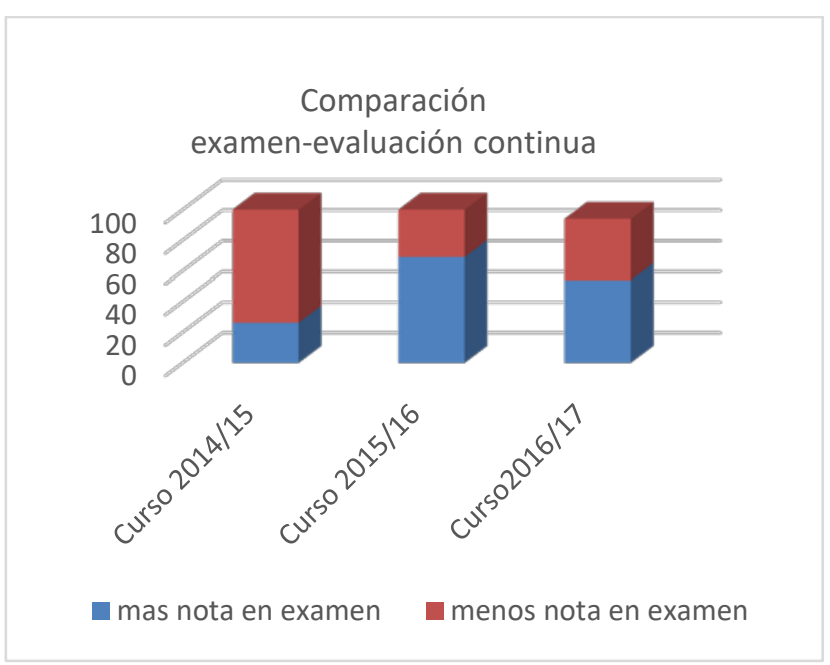

Figura 2: Notas examen-evaluación continua
Con la experiencia adquirida, en el próximo curso 20172018 podremos mejorar las rúbricas y realizar test para medir la satisfacción de cada una de las actividades realizadas por los alumnos y así continuar con nuestra tarea de introducir las mejoras docentes que sean necesarias.

\section{CONCLUSIONES}

En el trabajo aquí expuesto se quería mejorar la docencia de una asignatura del Grado de Diseño y Desarrollo de Videojuegos de forma que los alumnos entiendan la importancia de la misma en su titulación, a la vez que mejoren su rendimiento académico.

Con los resultados obtenidos hemos concluido que la realización de problemas, test y el uso de proyectos puede mejorar los resultados y adquisición de competencias de los alumnos.

Durante el presente curso hemos dado en las clases de teoría unas pautas a los alumnos para la búsqueda de la información que necesitaban para desarrollar su proyecto. Les hemos proporcionado un algoritmo para la realización en sus programas del módulo de comunicación entre "procesos", un cliente que se comunica con un servidor (que tiene el juego) o dos clientes que juegan entre sí un juego que ambos tienen.

Hemos desarrollado una serie de guiones en forma de "rúbricas" para que los alumnos tuvieran claro los pasos que debían ir siguiendo para acabar su proyecto de forma satisfactoria.

Creemos que esta experiencia es perfectamente transferible a cualquier asignatura. En nuestro caso hemos sustituido parte de las explicaciones teóricas por un trabajo experimental, los alumnos debían documentarse para realizar sus programas en java (lenguaje que la mayoría no conocía) utilizando primitivas de programación de redes, como son los sockets que vimos de forma escueta en clase de teoría.

Pensamos que se aprende más cuando uno tiene que realizar una labor de "investigación" sobre el tema que trabaja y se pelea mano a mano con los problemas que les van surgiendo.

Creemos que a los alumnos también les ha resultado una experiencia positiva. Nuestra intención es, una vez finalizado el plazo de evaluación, realizar una consulta para que ellos puedan proporcionarnos más datos al respecto.

\section{AGRADECIMIENTOS}

El trabajo aquí descrito se ha realizado gracias a la ayuda concedida por el proyecto docente con código 3208/16, "Proyecto de Innovación Educativa de la convocatoria 20162017 de la UJI, Unidad de Soporte Educativo (USE), Vicerrectorado de Estudiantes, Ocupación e Innovación Educativa.

\section{REFERENCIAS}

Cánovas Reverte, O., García Clemente, F.J. (2016). Prevención y seguimiento de factores limitantes del trabajo en equipo en experiencias ABP. En Actas de las XXII Jornadas de Enseñanza Universitaria de Informática, Jenui 2016, pp. 11 - 18.

Cuadrado Santolaria, R., Pérez Batle, M., Valero García, M. (2014). Controles de trabajo en grupo para mejorar la 
interdependencia positiva. En Actas de las XX Jornadas de Enseñanza Universitaria de Informática, Jenui 2014, pp. $363-370$.

Del Canto, P., Gallego, I., López, J.M., Mora, J., Reyes, A., Rodriguez, E., Sanjeevan, K., Santamaria, E., Valero, M. (2009). Conflictos en el trabajo en grupo: cuatro casos habituales. Revista de Formación e Innovación Educativa Universitaria. Vol.2. No 4, pp. 211-226.

Fernández Redondo, M., Hernández Espinosa, C.A., Torres Sospedra, J., Ramo Alegre, P. (2009). "Diseño de nuevas prácticas de laboratorio para la adaptación a la actual asignatura armonizada de Redes (IS20) de la Ingeniería Técnica en Informática de Sistemas”. Experiencias de mejora e innovación educativa de la Universidad Jaume I, curso 2008-2009, Actas de la IX Jornada de Mejora Educativa de la UJI, pp. 127-132.

Fernández Redondo, M., Hernández Espinosa, C.A., Torres Sospedra, J., Ramo Alegre, P. (2009). "Diseño de sistemas de autoevaluación para la adecuación a los créditos ECTS de la asignatura Redes (IS20) de la Ingeniería Técnica en Informática de Sistemas”. Experiencias de mejora e innovación educativa de la Universidad Jaume I, curso 2008-2009, Actas de la IX Jornada de Mejora Educativa de la UJI, pp. 69-74.

Fernández Redondo, M., Hernández Espinosa, C.A., Recatalá Ballester, G., Sales Gil, J. (2013). "Desarrollo de actividades de autoaprendizaje y cambio de metodología docente en asignaturas no presenciales o presenciales basadas en problemas y proyectos”. II Congreso
Internacional sobre Aprendizaje, Innovación y Competitividad (CINAIC 2013).

Fernández Redondo, M., Hernández Espinosa, C.A., Torres Sospedra, J. (2009) "Diseño de sistemas de autoevaluación para la adecuación a los créditos ECTS de la asignatura Redes (IS20) de la Ingeniería Técnica en Informática de Sistemas”. Experiencias de mejora $e$ innovación educativa de la Universidad Jaume I, curso 2008-2009, Actas de la IX Jornada de Mejora Educativa de la UJI, pp. 69-74.

López, D., Carrera, D. (2016) Viabilidad y escalabilidad de actividades substitutivas del examen: un caso práctico. En Actas de las XXII Jornadas de Enseñanza Universitaria de Informática, Jenui 2016, pp. 61 - 68.

Mollineda Cárdenas, R.A. (2012). Aprendizaje basado en problemas y en comparación de soluciones en un contexto semipresencial. En Actas de las XVIII Jornadas de Enseñanza Universitaria de Informática, Jenui 2012, pp. $373-376$.

Oliver, J. (2016). Desarrollo multinivel de la competencia transversal de trabajo en equipo. En Actas de las XXII Jornadas de Enseñanza Universitaria de Informática, Jenui 2016, pp. 6 - 8.

Sebastian, R., Olanda, R., Orduña, J.M. (2013). Introducción de metodologías de aprendizaje basado en problemas en el marco de las TIC. En Actas de las XIX Jornadas de Enseñanza Universitaria de Informática, Jenui 2013, pp. 153

160 